\title{
Thermopower oscillations in mesoscopic Andreev interferometers
}

\author{
M. Titov \\ School of Engineering \& Physical Sciences, Heriot-Watt University, Edinburgh EH14 4AS, UK
}

(Dated: August, 2008)

\begin{abstract}
Anomalously large thermopower of mesoscopic normal-metal/superconductor interferometers has been investigated by Chandrasekhar et al. It was shown that, depending on the geometry of the interferometer, the thermopower is either symmetric or antisymmetric periodic function of the magnetic flux. We develop a detailed theory of the observed thermoelectric phenomena in the framework of the non-equilibrium quasiclassical approach. In particular we provide, for the first time, a possible explanation of the symmetric thermopower oscillations. This effect is attributed to the electron-hole symmetry violation that originates in the steady-state charge imbalance between different arms of the interferometer. Our theory can be tested by an additional control over the charge imbalance in a modified setup geometry. We also predict a sign reversal behavior of the thermopower with increasing temperature that is consistent with the experiments by Parsons et al.

PACS numbers: 74.45.+c,74.25.Fy, 73.23.-b
\end{abstract}

\section{INTRODUCTION}

A linear response of a metal to the temperature gradient $\nabla T$ and external electric field $E$ is characterized by four transport coefficients: $\sigma, \kappa, \eta, \zeta$, which are defined, under the open circuit conditions, by the following relation 1

$$
\left(\begin{array}{l}
\boldsymbol{J}^{e} \\
\boldsymbol{J}^{Q}
\end{array}\right)=\left(\begin{array}{ll}
\sigma & \eta \\
\zeta & \kappa
\end{array}\right)\left(\begin{array}{c}
\mathcal{E} \\
-\nabla T
\end{array}\right)
$$

where $\boldsymbol{J}^{e}$ is the electric current and $\boldsymbol{J}^{Q}$ is the heat flow induced in the circuit. The diagonal terms, $\sigma$ and $\kappa$, are the electric and thermal conductivity, while the off-diagonal ones, $\eta$ and $\zeta$, are the thermoelectric coefficients that fulfill the Onsager relation $\zeta=T \eta$.

The thermoelectric response is usually observed in a confined geometry by measuring the voltage rather than the electric current. The ratio of the measured voltage to the temperature gradient applied across the sample is known as the Seebeck coefficient (or, the thermopower) and is given by $S=\eta / \sigma=\left.(\mathcal{E} / \nabla T)\right|_{J^{e}=0}$. The Mott's formula ${ }^{2}$ relates the thermopower to the energy dependent conductivity $\sigma(\varepsilon)$

$$
S=-\left.\frac{\pi^{2}}{3} \frac{k_{B}^{2} T}{e} \frac{d \ln \sigma(\varepsilon)}{d \varepsilon}\right|_{\varepsilon=\varepsilon_{F}},
$$

and illustrates an important role of the electron-hole asymmetry for the thermoelectric response in metals. Indeed, one can see from Eq. (2) that $S$ vanishes if $\sigma(\varepsilon)$ is symmetric around the Fermi energy $\varepsilon_{F}$. In the case of a spherical Fermi surface the thermopower $S \sim k_{B}^{2} T / e \varepsilon_{F}$ is finite only because of a tiny difference in the effective masses of electrons and holes.

In superconductors the situation is complicated by the presence of Bose condensate of Cooper pairs that does not respond to a temperature gradient. It was suggested by Ginzburg ${ }^{3}$ on the basis of the two-fluid model of superconductivity that the linear response theory (1) still applies to a normal (dissipative) component of the electron liquid. The thermoelectric effect is, however, shunted by the superconducting component and cannot be observed in bulk superconductors. 4.5 An incomplete cancellation of the thermoelectric and supercurrents was, nevertheless, predicted for anisotropic superconductors and superconducting bimetallic samples. ${ }^{6,7}$ This theory has been experimentally confirmed by Zavaritskii $\stackrel{-}{-}$ by using a bimetallic loop to detect the thermopower in a superconducting state. The method relies upon the quantization of the superconducting condensate in the loop that prevents a complete cancellation of the thermoelectric current and generates a small, but measurable, magnetic flux $\Phi_{T} \sim 10^{-2} \Phi_{0}$, where $\Phi_{0}=2 e / h$ is the flux quantum. In subsequent experiments, ${ }^{9}$ however, much greater values of the thermoelectrically induced flux, $\Phi_{T} \sim 10^{2} \Phi_{0}$, were detected. The origin of this "giant flux" puzzle remains debated..$^{10,11}$

Mesoscopic systems provide an altogether different way to probe the thermoelectric phenomena in the presence of superconductivity. The superconducting correlations can penetrate the normal-metal part of the system due to a process known as Andreev reflection. As the result the thermopower of a mesoscopic normal-metal wire can be affected by contacting the wire to a superconductor. First experiments of this kind have been performed a decade ago by Chandrasekhar et al. ${ }^{12}$ (see Fig. 1). In these and in numerous subsequent experiments $13,14,15,16,17,18,19,20,21$ the thermopower of a normal-metal wire in proximity to a superconducor/normalmetal loop was found to oscillate as a function of the magnetic flux piercing the loop. The amplitude of the oscillations was shown to exceed the thermopower of the normalmetal wire in the absence of proximity-induced superconductivity. The experimental results suggest that the proximity effect is responsible for the electron-hole symmetry violation in these systems, which is no longer suppressed by a small factor $k_{B} T / \varepsilon_{F}$. The lack of the electron-hole symmetry in the experiments was attributed long ago ${ }^{22,23}$ to a voltage induced between the superconducting and the normal-metal part of the mesoscopic circuit under non-equilibrium conditions. This voltage is directly related to the shift between the chemical potential in the normal metal and that in the superconductor. Similar shift, which is commonly referred to as the charge imbalance, can exist inside a bulk superconductor between the chemical potential of quasiparticles and that of the Cooper pairs.

The charge imbalance in bulk superconductors and its 
role in the thermoelectric effects were intensely studied in 1970s (see Ref. 24 for the review.). The imbalance can be achieved either by injection of a dissipative current to the superconductor $25,26,27,28$ or by applying a temperature gradient in the presence of a superconducting flow ${ }^{29,30,31}$ (PethickSmith effect). The relaxation of the charge imbalance is determined primarily by the electron-phonon interaction. From the detailed theory provided by Schmid and Schön ${ }^{32,33}$ one can roughly estimate the imbalance relaxation time in bulk $s$-wave superconductors as $\tau_{Q} \sim\left(k_{B} T / \Delta\right) \tau_{i n}$, where $\Delta$ is the value of the superconducting order parameter and $\tau_{i n}$ is the temperature dependent electron-phonon scattering time. In our study we deal instead with the voltage drop between the superconducting and the normal-metal arms of the interferometer. We, however, keep using the term imbalance to stress the analogy to the thermoelectric effects in bulk superconductors.

The non-equilibrium quasiclassical theory of proximity effect has been successfully applied in Refs. 34, 35, 36, 37 to describe the antisymmetric magnetic field dependence of the thermopower in Fig. 1. In this paper we develop a general analytical approach that explains both antisymmetric and symmetric dependence of the thermopower observed in the experiment. Our theory is based on the quasiclassical kinetic equations in the limit of weak proximity effect. Even though this approximation assumes a small transparency of the normalmetal/superconductor interfaces in Fig. 2, it does not affect the symmetry of the obtained results.

In the Section IV we solve the kinetic equations for the parallelogram interferometer depicted schematically in Fig. 3 In accordance with earlier studies we find that the charging of the superconducting arm of the interferometer is determined by the interplay of the supercurrent and the temperature gradient. The formation of the charge imbalance is analogous to that in the Pethick-Smith effect. ${ }^{29}$ The extra charge accumulated by the superconductor is, therefore, a purely antisymmetric function of both the temperature gradient and the applied magnetic flux. In this case the left-right symmetry of the structure has to be broken in order to observe the effect of proximity-induced correlations on the thermopower of the normal-metal wire 34 The symmetry breaking can be caused either by a difference between $L_{1}$ and $L_{2}$ in Fig. 3 assuming that these distances are much smaller than the phase-coherence length $L_{\phi}$, or by a difference in the $N S$ interface transparencies $\alpha_{1}$ and $\alpha_{2}$, which are given by the ratio of the normal wire resistance per unit length to the interface resistance. In the latter case the phase coherence of quasiparticles in the normal-metal wire is irrelevant for the symmetry breaking. We also stress that the energy relaxation processes in the superconductor play an important role in the theory of the thermoelectric effect. Indeed, the time-independent solution to the kinetic problem exists only if the detailed balance condition at a given energy is broken. Another words, in the steady-state limit, only the total charge transmitted through the superconducting wire is conserved while the energy density of the charge flow is not. The steady-state regime develops at times exceeding the imbalance relaxation time $\tau_{Q}$ inside the superconducting wire. The resulting behavior of the thermopower in the parallelogram interferometer is illustrated in Fig. 4 as a function of temperature.

In the Section $\mathrm{V}$ we apply the same approach to the house interferometer, which is shown schematically in Fig. 5. In sharp contrast to the previous case we find a finite contribution to the thermopower in the absence of a charge imbalance between the normal-metal and the superconducting branches. Such contribution has an antisymmetric dependence on the magnetic flux and requires a left-right asymmetry of the wire $N^{\prime}$ that must originate in a difference between $d_{1}$ and $d_{2}$ rather than in a difference between the $N S$ interface transparencies $\tilde{\alpha}_{1}$ and $\tilde{\alpha}_{2}$. This asymmetric dependence of the thermopower in the house interferometer is a phase-coherent phenomena that can be attributed to the interference of quasiparticle trajectories shown in Fig. 6. Apart from the evident requirement $d_{1}, d_{2} \ll L_{\phi}$ this effect is strongly sensitive to the position of the Andreev reflection. ${ }^{38}$ In the parallelogram interferometer the interference contribution to the thermopower is averaged to zero since it involves the summation over the trajectories of different lengths.

The odd oscillations of the thermopower are, however, not seen in experiments with the house interferometer. Instead, a sign-definite thermopower, which has an even dependence on the magnetic flux $\Phi$, has been observed. 12,16,21 Moreover, the maximal values of the symmetric thermopower in Fig. $1 \mathrm{~b}$ correspond to $\Phi=n \Phi_{0}$, where $n$ is an integer number and $\Phi_{0}=h / 2 e$ is the flux quantum. We demonstrate that this behavior is a signature of the finite charge imbalance maintained between the Cooper-pair chemical potential in the superconductor and the quasiparticle chemical potential in the normalmetal. Our theory predicts that the thermopower in this case is proportional to $\mu\left(1+\cos 2 \pi \Phi / \Phi_{0}\right)$, hence its magnetic field dependence agrees with the experimental data ${ }^{12}$ in Fig $1 b$. The thermopower determined by this effect has a peculiar signreversal behavior with increasing temperature that is illustrated in Fig. 7 This temperature dependence is in qualitative agreement with the experiments by Parsons et al. ${ }^{16}$

To our opinion the experiments of Refs. 12, 16, 21 provide a clear signature of a finite steady-state imbalance $\mu$ in the house interferometer. We demonstrate that a tiny chemical potential imbalance between different branches of the house interferometer has a great impact on the thermopower due to the proximity effect. The possible origin of the tiny imbalance is in the usual thermoelectric effect caused by a heat dissipation in the second normal-metal wire $N^{\prime}$. The validity of this scenario can be tested by varying the chemical potential in $N^{\prime}$ with the help of an additional electrode or by a detailed measurement of the temperature dependence of the thermopower in the house interferometer.

The remainder of the paper is organized as follows. In the Section $\amalg$ we introduce the well-known quasiclassical approach to the proximity effects in diffusive mesoscopic wires. In the Section $\amalg$ the rigid boundary conditions at a three terminal contact are given. The Sections IV $\mathrm{V}$ are devoted to the analytical calculation of the thermopower for the parallelogram and the house interferometer, correspondingly. We summarize our conclusions in the SectionVI 

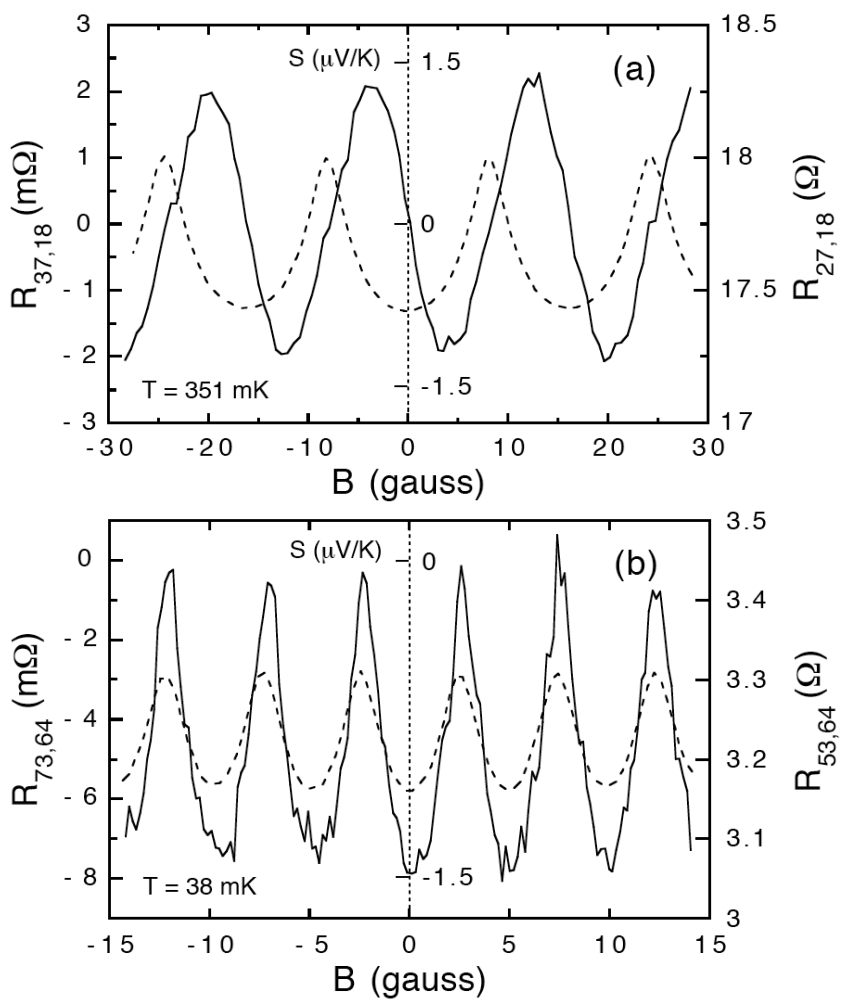

FIG. 1: The experimentally observed thermopower oscillations (solid line) in the parallelogram (a) and in the house (b) interferometer. The figure is adopted from Ref. 12 .

\section{QUASICLASSICAL THEORY}

Andreev reflection is the main microscopic mechanism of charge transport between the superconductor (S) and the normal-metal $(\mathrm{N})$ at low temperatures. It can be seen from the normal-metal side as the conversion of electron-like excitations of the energy $\varepsilon$ to the hole-like ones. The reflection takes place at the $N S$ boundary and is responsible for the phase coherence of electrons and holes at a distance $\sqrt{D / \varepsilon}$ in the diffusive normal metal, where $D$ is the diffusion coefficient (we set $\hbar=k_{B}=1$ throughout the rest of the paper). From the superconductor side the same process can be viewed as a diffusion of Cooper pairs that brings the superconducting correlations into the normal metal.

The characteristic scale of the proximity effect at a temperature $T$ is determined by the coherence length $\xi=\sqrt{D / T}$. The electron-hole coherence can be detected in the diffusive metal provided $T \lesssim E_{c}$, where $E_{c}=D / L^{2}$ is the Thouless energy associated with the distance $L$ to the $N S$ interface. On the ballistic scales, $E_{c}>\Delta$, the role of $E_{c}$ is played by $\Delta$, the absolute value of the superconductor energy gap.

The conditions for the diffusive proximity effect were fulfilled in the pioneering experiments with Andreev interferometers performed by Chandrasekhar et al. ${ }^{12}$ and Parsons et al. ${ }^{16}$ We, therefore, reduce our consideration to the diffusive approximation of the quasiclassical theory that is described by the Usadel equation.
We shall start from the stationary Usadel equation written for the quasiclassical Green's function $\check{g}(x, \varepsilon)$ in a diffusive normal-metal wire,

$$
D \frac{d \check{I}}{d x}+\left[i \varepsilon \sigma_{z}, \check{g}\right]=0, \quad \check{I} \equiv \check{g} \frac{d \check{g}}{d x},
$$

where $D$ is the diffusion coefficient, $\check{I}$ is the matrix current, and $x \in\left(-L_{1}, L_{2}\right)$ is the coordinate along the normal-metal wire. The Green's function is represented by a matrix in the Keldysh space,

$$
\check{g}=\left(\begin{array}{cc}
\hat{g}^{R} & \hat{g}^{K} \\
0 & \hat{g}^{A}
\end{array}\right), \quad \check{g}^{2}=1,
$$

which yields the quasiclassical constraint $\check{g}^{2}=1$.

Despite the absence of the superconducting order parameter in the metallic wire, the proximity effect will lead to a superconducting correlations that are described by the anomalous components $f^{R, A}$ of the Green's function. These correlations are taken into account by the extension to the electronhole space. The spectral (retarded and advanced) sector of the Green's function in this space is parameterized as

$$
\hat{g}^{R(A)}=\left(\begin{array}{cc} 
\pm g^{R(A)} & f^{R(A)} \\
\bar{f}^{R(A)} & \mp g^{R(A)}
\end{array}\right),
$$

where bar stands for the charge conjugation. The relation

$$
g^{R(A)}=\sqrt{1-f^{R(A)} \bar{f}^{R(A)}},
$$

follows from the quasiclassical constraint (4). The Keldysh component of the constraint, $\hat{g}^{R} \hat{g}^{K}+\hat{g}^{K} \hat{g}^{A}=0$, suggests that $\hat{g}^{K}$ has only two linearly independent entities. In what follows we use the standard parameterization

$$
\hat{g}^{K}=\hat{g}^{R} \hat{h}-\hat{h} \hat{g}^{A},
$$

where the diagonal matrix

$$
\hat{h}=h+\sigma_{z} h_{\sigma},
$$

has two degrees of freedom: $h$ and $h_{\sigma}$.

We model the experimental situation by an idealized system consisting of two equilibrium reservoirs connected by a noninteracting normal-metal diffusive wire $N$. The distribution functions $\hat{h}_{1}$ and $\hat{h}_{2}$ in the reservoirs are parameterized by the chemical potentials $\mu_{1}, \mu_{2}$ and the temperatures $T_{1}, T_{2}$ as

$$
\left.\hat{h}\right|_{x \rightarrow-L_{1}, L_{2}}=\left(\begin{array}{cc}
h_{1,2} & 0 \\
0 & \bar{h}_{1,2}
\end{array}\right) \text {, }
$$

where

$$
h_{a}=\tanh \frac{\varepsilon-\mu_{a}}{2 T_{a}}, \quad \bar{h}_{a}=\tanh \frac{\varepsilon+\mu_{a}}{2 T_{a}},
$$

and $a=1,2$. The functions $\left(1-h_{a}\right) / 2,\left(1-\bar{h}_{a}\right) / 2$ are the Fermi distribution functions of electrons and holes, correspondingly. The electric and the heat current in the normalmetal wire are obtained in the Usadel approximation from the 
Keldysh component of the matrix current $\check{I}$,

$$
\begin{array}{ll}
J^{e}=\frac{e D \nu}{4} \int d \varepsilon j^{e}(\varepsilon), & j^{e}(\varepsilon)=\operatorname{Tr} \sigma_{z} \hat{I}^{K}, \\
J^{Q}=\frac{D \nu}{4} \int d \varepsilon \varepsilon j^{Q}(\varepsilon), & j^{Q}(\varepsilon)=\operatorname{Tr} \hat{I}^{K},
\end{array}
$$

where $\hat{I}^{K}=\hat{g}^{R}\left(d \hat{g}^{K} / d x\right)+\hat{g}^{K}\left(d \hat{g}^{A} / d x\right)$.

The Usadel equation (3) does not take into account inelastic processes, hence the charge and heat flow is conserved for each energy. Indeed, from the diagonal components of its Keldysh sector we obtain the conservation laws

$$
\frac{d j^{e}(\varepsilon)}{d x}=\frac{d j^{Q}(\varepsilon)}{d x}=0,
$$

which play a role of the kinetic equation in the normal-metal wire. The diagonal components of the retarded and advanced component of the Usadel equation (3) imply the conservation of the "spectral" current

$$
\frac{d W^{R}}{d x}=\frac{d W^{A}}{d x}=0
$$

where $W^{R}=f^{R}\left(d \bar{f}^{R} / d x\right)-\bar{f}^{R}\left(d f^{R} / d x\right)$. This condition is equivalent to the conservation of the supercurrent, which can flow in the normal metal in the presence of the proximity effect.

From Eqs. (11) we find the current energy densities $j^{e}(\varepsilon)$, $j^{Q}(\varepsilon)$ in the parameterization of Eqs. (517) as

$$
\begin{aligned}
& j^{e}(\varepsilon)=M_{+} \frac{d h_{\sigma}}{d x}-U \frac{d h}{d x}+W h, \\
& j^{Q}(\varepsilon)=M_{-} \frac{d h}{d x}+U \frac{d h_{\sigma}}{d x}+W h_{\sigma},
\end{aligned}
$$

where the following functions, which depend on the energy and coordinate, are introduced

$$
\begin{aligned}
& M_{ \pm}=\left(2\left(1+g^{R} g^{A}\right) \pm\left(f^{R} \bar{f}^{A}+f^{A} \bar{f}^{R}\right)\right) \\
& U=f^{R} \bar{f}^{A}-f^{A} \bar{f}^{R}, \quad W=W^{R}-W^{A}
\end{aligned}
$$

Thus, the kinetic part of the transport problem is fully described by Eqs. (1214).

It is instructive to apply this formalism to the transport in a metallic wire in the absence of a proximity effect. In this case one finds $f^{R(A)}=0$, hence $g^{R}=-g^{A}=\sigma_{z}$ and

$$
j^{e}=4 \frac{d h_{\sigma}}{d x}, \quad j^{Q}=4 \frac{d h}{d x} .
$$

The solution to Eqs. (12), which yields the boundary conditions (9), reads

$$
\begin{aligned}
h(\varepsilon, x) & =\frac{1}{4}\left(h_{0}+\frac{L_{2}-L_{1}-2 x}{L} h_{T}\right), \\
h_{\sigma}(\varepsilon, x) & =\frac{1}{4}\left(h_{\mu}+\frac{L_{2}-L_{1}-2 x}{L} h_{\mu T}\right),
\end{aligned}
$$

where the following notations are used

$$
\begin{aligned}
h_{0} & =h_{1}+\bar{h}_{1}+h_{2}+\bar{h}_{2}, \\
h_{T} & =h_{1}+\bar{h}_{1}-h_{2}-\bar{h}_{2}, \\
h_{\mu} & =h_{1}-\bar{h}_{1}+h_{2}-\bar{h}_{2}, \\
h_{\mu T} & =h_{1}-\bar{h}_{1}-h_{2}+\bar{h}_{2} .
\end{aligned}
$$

Substituting Eqs. (17) to Eqs. (16) we obtain $j^{e}=$ $-(2 / L) h_{\mu T}$ and $j^{Q}=-(2 / L) h_{T}$.

If a temperature gradient is applied between the reservoirs that are kept at the same chemical potential $\mu=\mu_{1}=\mu_{2}$, the integration in Eq. (11a) gives $J^{e}=0$, hence $\eta=0$. The heat current in the limit $\left|T_{1}-T_{2}\right| \ll T$ is given by Eq. (11b) as

$$
J^{Q}=-\kappa\left(T_{2}-T_{1}\right), \quad \kappa=\frac{\pi^{2}}{3} \frac{2 D \nu T}{L},
$$

where $T=\left(T_{1}+T_{2}\right) / 2$ is the mean temperature in the system and $\kappa$ is the thermal conductivity.

Similarly, if the chemical potential difference is applied between the reservoirs that are kept at the same temperature $T$, the integration in Eqs. (11) gives $j^{Q}=0$ and

$$
J^{e}=\sigma \frac{\mu_{2}-\mu_{1}}{(-e) L}, \quad \sigma=2 e^{2} D \nu
$$

where $\sigma$ is the Drude result for the electric conductivity.

We shall stress that the vanishing of the thermoelectric coefficients $\eta$ and $\zeta$ in the calculation above is a consequence of the linearization of the quasiparticle spectrum near the Fermi energy $\varepsilon_{F}$. Such a linearization is essential for the quasiclassical approximation and leads to the exact electron-hole symmetry in metals. The proximity effect can, however, break this symmetry through the possible energy dependence of $M_{ \pm}, U$ and $W$ in Eq. (14) that can give rise to a finite thermoelectric response in the system. Such thermoelectric phenomena can be analyzed within the quasiclassical approximation.

Since the superconducting arm of the interferometer used in experiments exceeds the electron-phonon scattering length it can be described by the equilibrium quasiclassical Green's function $\check{g}_{S}$. We assume for simplicity that the superconducting energy gap drops abruptly to zero in the normalmetal/superconductor interface. Even though this assumption is not self-consistent and disregards a small suppression of $\Delta$ near the $N S$ boundary, it does not affect our conclusions on the symmetry of the thermopower oscillations.

We, therefore, describe the superconductor by the standard bulk expressions for the spectral components of $\check{g}_{S}$ that are given by

$$
\hat{g}_{S}^{R(A)}=\frac{1}{\sqrt{(0 \mp i \varepsilon)^{2}+\Delta^{2}}}\left(\begin{array}{cc}
-i \varepsilon & \Delta e^{i \chi} \\
\Delta e^{-i \chi} & i \varepsilon
\end{array}\right),
$$

where $\Delta \exp (i \chi)$ is the superconducting order parameter.

The external magnetic flux $\Phi$ piercing the loop leads to a gradient of the phase $\chi$, hence the phase acquires different values $\chi_{1}$ and $\chi_{2}$ at the opposite ends of the superconducting wire. The order parameter phase difference,

$$
\varphi=\chi_{1}-\chi_{2}
$$


is related to the magnetic flux as $\varphi=2 \pi \Phi / \Phi_{0}$. The Keldysh component of $\check{g}_{S}$ is parameterized by

$$
\hat{g}_{S}^{K}=\left(\hat{g}_{S}^{R}-\hat{g}_{S}^{A}\right) h_{S},
$$

where $\hat{h}_{S}=\tanh \left(\varepsilon / 2 T_{S}\right)$ is the quasiparticle distribution function and $T_{S}$ is the temperature of the superconductor. Here we choose the chemical potential of the Cooper pairs as zero and set $h_{S \sigma}=0$. For $\varepsilon<\Delta$ one finds from Eq. (21) that $\hat{g}_{S}^{R}=\hat{g}_{S}^{A}$, hence $\hat{g}_{S}^{K}=0$. In this paper we are concerned with ultra low temperatures $T \ll \Delta$ and, therefore, disregard all quasiparticle effects in the superconductor.

The effective energy scale for the proximity effect in the normal metal is given by the Thouless energy $E_{c}=D / L^{2}$, where $L$ is the distance from the $N S$ interface. To describe the experimentally relevant situation $T \sim E_{c} \ll \Delta$, we restrict our consideration to the excitation energies that are much smaller than the absolute value of the superconducting gap, therefore

$$
\hat{g}_{S}^{R}=\hat{g}_{S}^{A}=\left(\begin{array}{cc}
0 & e^{i \chi} \\
e^{-i \chi} & 0
\end{array}\right) .
$$

We will see below that our results for the thermopower are indeed defined by small excitation energies $\varepsilon \leq E_{c}$, which proves the the consistency of this approximation.

In addition we assume that the proximity effect is weak due to a low transparency of the NS interfaces. This assumption allows for the linearization of the Usadel equation (3) with respect to the anomalous component of the Green's function,

$$
\frac{d^{2} f^{R}}{d x^{2}}=z^{2} f^{R}, \quad z^{2} \equiv \frac{2(0-i \varepsilon)}{D},
$$

where we replace $\varepsilon$ by $\varepsilon-i 0$ in order to stress the analytical properties of $f^{R}$. The solution for the advanced Green's function is obtained by the substitution $\varepsilon \rightarrow-\varepsilon$. The conservation of the supercurrent in Eq. (13) is equivalent in this approximation to the standard property of the Wronskian of a linear second-order differential equation. The following symmetry relations simplify the subsequent analysis,

$$
f^{A}(\varepsilon)=f^{R}(-\varepsilon), \bar{f}^{R}(\Phi)=f^{R}(-\Phi), \bar{f}^{A}=\left(f^{R}\right)^{*} .
$$

\section{BOUNDARY CONDITIONS}

The boundary condition for a junction shown in Fig. 2 is an important ingredient of our description of the proximity effect in Andreev interferometers. We assume that a contact wire, which is either a superconductor in the parallelogram interferometer or a normal-metal wire $N^{\prime}$ in the house interferometer, is described by the Green's function $\check{g}_{c}$. The Green's function in the main wire $N$ is denoted by $\check{g}$.

The transparency of the barrier between the contact and the normal-metal wire is parameterized by a coefficient $\alpha=$ $T_{B} / \ell_{B}$. It has a dimension of an inverse length, where $T_{B}$ is the barrier transmission probability per channel and $\ell_{B}$ is an effective barrier length, which is of the order of the mean free

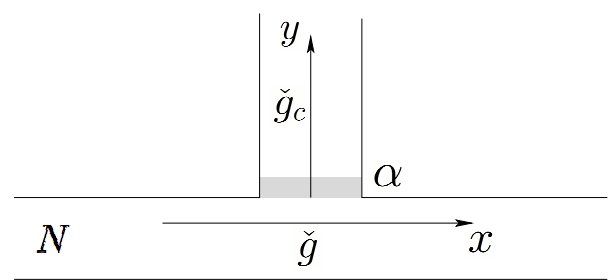

FIG. 2: The three-terminal junction formed by a contact attached to the main normal-metal wire $N$. The generalized rigid boundary conditions at the junction are given by Eqs. 27/28 in the matrix notation. The Green's function $\check{g}$ is continuous along the wire $N$ but there is a jump in the Green's function across the tunnel barrier so that $\check{g} \neq \check{g}$ at the junction. The kinetic component of Eq. 28) is equivalent to Eqs. 30[31.

path in $N$. The coefficient $\alpha$ can also be regarded as the ratio of the normal-wire resistance per unit length to the interface resistance and has a dimension of an inverse length.

The generalized "rigid" boundary conditions ${ }^{39}$ apply provided $T_{B} \ll 1$ for each transmission channel at the junction. We stress that the tunnel barrier in Fig. 2 is placed between the normal-metal wire $N$ and the contact wire. As the result the green's function $\check{g}$ is continuous along the normal metal wire while its derivative has a discontinuity that is determined by a difference between $\breve{g}$ and $\check{g}_{c}$. The continuity of the Green's function in the $N$ wire is formally written as

$$
\delta[\check{g}]_{x_{0}} \equiv \lim _{\delta \rightarrow 0}\left(\check{g}\left(x_{0}+\delta\right)-\check{g}\left(x_{0}-\delta\right)\right)=0 .
$$

The discontinuity of the derivative of $\check{g}(x)$ is obtained from the matrix current conservation in the three terminal junction

$$
\delta[\check{I}]_{x_{0}}=\left.\frac{\alpha}{2}\left[\check{g}_{c}, \check{g}\right]\right|_{x_{0}} .
$$

Note, that the green's function is discontinuous across the tunnel barrier, i.e. $\check{g} \neq \check{g}_{c}$ at $x=x_{0}$. One can easily demonstrate that Eq. (28) is a discrete analog of the Usadel equation (3) and yields the same set of conservation laws.

For instance, the spectral part of Eqs. (27/28) leads to

$$
\begin{aligned}
& \delta[W]_{x_{0}}=\tilde{W}=\tilde{W}^{R}-\tilde{W}^{A}, \\
& \tilde{W}^{R}=-\alpha\left(f^{R} \bar{f}_{c}^{R}-f_{c}^{R} \bar{f}^{R}\right),
\end{aligned}
$$

which illustrates the conservation of the suppercurrent at the junction.

In a full analogy with Eqs. (12/14) the Keldysh sector of Eqs. (27/28) is given by

$$
\begin{aligned}
& \delta\left[j^{e}(\varepsilon)\right]_{x_{0}}=\frac{\alpha}{2} \operatorname{Tr} \sigma_{z}\left[\check{g}_{c}, \check{g}\right]_{x_{0}}^{K} \\
& =\alpha\left(h_{\sigma}-h_{c \sigma}\right) \tilde{M}_{+}-\alpha\left(h-h_{c}\right) \tilde{U}+\frac{h+h_{c}}{2} \tilde{W}, \\
& \delta\left[j^{Q}(\varepsilon)\right]_{x_{0}}=\frac{\alpha}{2} \operatorname{Tr}\left[\check{g}_{c}, \check{g}_{x_{0}}^{K}\right. \\
& \quad=\alpha\left(h-h_{c}\right) \tilde{M}_{-}+\alpha\left(h_{\sigma}-h_{c \sigma}\right) \tilde{U}+\frac{h_{\sigma}+h_{\sigma c}}{2} \tilde{W},
\end{aligned}
$$




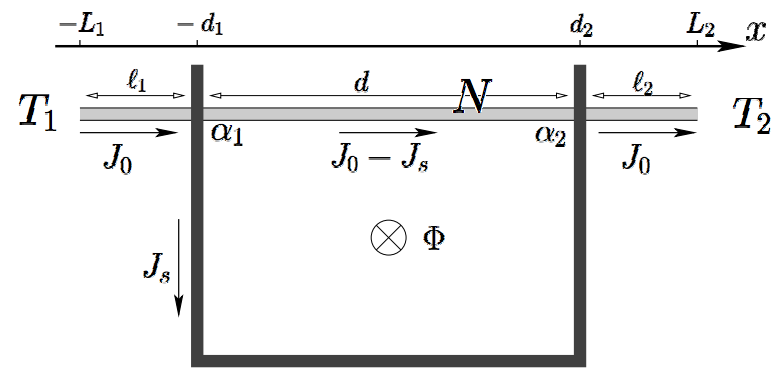

FIG. 3: The parallelogram interferometer. Diffusive normal-metal wire $N$ of the length $L=L_{1}+L_{2}$ connects the temperature reservoirs denoted as $T_{1}$ and $T_{2}$. The superconducting wire (dark line) is contacting $N$ at two junctions separated by a distance $d$. The $N S$ interfaces are characterized by the transparency parameters $\alpha_{1}$ and $\alpha_{2}$. Only antisymmetric dependence of the thermopower on the magnetic flux is allowed by symmetry. The effect requires $\ell_{1} \neq \ell_{2}$ or $\alpha_{1} \neq \alpha_{2}$.

where the following notations are introduced

$$
\begin{aligned}
\tilde{M}_{ \pm}= & \left(g^{R}+g^{A}\right)\left(g_{c}^{R}+g_{c}^{A}\right)+\frac{1}{2}\left(f^{R}+f^{A}\right)\left(\bar{f}_{c}^{R}+\bar{f}_{c}^{A}\right) \\
& +\frac{1}{2}\left(\bar{f}^{R}+\bar{f}^{A}\right)\left(f_{c}^{R}+f_{c}^{A}\right) \\
\tilde{U}= & \frac{1}{2}\left(f^{R} \bar{f}_{c}^{A}-\bar{f}^{R} f_{c}^{A}-f^{A} \bar{f}_{c}^{R}+\bar{f}^{A} f_{c}^{R}\right) \\
\tilde{W}= & -\alpha\left(f^{R} \bar{f}_{c}^{R}-\bar{f}^{R} f_{c}^{R}-f^{A} \bar{f}_{c}^{A}+\bar{f}^{A} f_{c}^{A}\right)
\end{aligned}
$$

The Equations (30|31) relate the discontinuity in the current in the normal-metal wire $N$ to the current flowing into the contact wire.

\section{THERMOPOWER IN THE PARALLELOGRAM INTERFEROMETER}

The parallelogram interferometer realized in the experiment of Ref. 12 is depicted schematically in Fig. 3, The thermopower is measured between the temperature reservoirs $T_{1}$ and $T_{2}$ that are connected by the normal-metal wire $N(\mathrm{Au})$ of the length $L=L_{1}+L_{2} \sim 1.7 \mu \mathrm{m}$. The wire is contacted to a long superconducting arm at the points $x=-d_{1}$ and $x=d_{2}$ with a distance $d=d_{1}+d_{2} \sim 0.2 \mu \mathrm{m}$ between them. The thermopower measurement in Fig. 11 is performed at the temperature $T=350 \mathrm{mK}$. The superconducting coherence length at this temperature is estimated as $\xi=\sqrt{D / T} \sim 0.54 \mu \mathrm{m}$, while the phase coherence length is approximately given by $L_{\phi} \sim 3.5 \mu \mathrm{m}$. Thus, the experimental parameters are such that $d \lesssim \xi$. Therefore, if an external magnetic flux $\Phi$ is piercing the interferometer, the supercurrent $J_{s}$ flows in the normal-metal wire between the $N S$ junctions.

In the subsequent analysis we let $\mu_{1}=\mu_{2}=\mu$ and calculate the quasiparticle current $J_{0}$ between the reservoirs to the first order in the temperature difference $T_{1}-T_{2}$, thus, obtaining the coefficient $\eta$. The thermopower is, then, calculated from the relation $S=\eta / \sigma$. In the limit of weak proximity effect the Drude result for $\sigma$ can be used. We note that a similar approach has been developed in the series of publications by Virtanen and Heikilla. $34,35,36$

In order to keep our formulas compact we define the distances $\ell_{1}$ and $\ell_{2}$ between the $N S$ interfaces and the corresponding reservoirs as $\ell_{a} \equiv L_{a}-d_{a}$, where $a=1,2$. The important simplification, which enables us to solve the problem analytically, is the linearization of the Usadel equations (25) for the spectral components of the Green's function $\check{g}(x)$. This approximation is justified since the wire is in a good contact to the reservoirs with a vanishing proximity effect,

$$
\left.f^{R(A)}\right|_{x=-L_{1}}=\left.f^{R(A)}\right|_{x=L_{2}}=0 .
$$

We remind that the chemical potential $\mu$ in the reservoirs is measured with respect to that of the Cooper pairs in the superconductor, therefore, the temperature equilibrium is characterized by $\mu=0$.

We start by pointing out that the energy relaxation processes are important for the existence of a steady-state regime with a finite $\mu$ in the parallelogram interferometer. Indeed, if scattering is purely elastic the charge conservation law in the supercondutor must hold for each energy. In this case the time-independent solution to the kinetic equation does not exist. Following other works ${ }^{22.34}$ we relax the energy-resolved condition to the conservation of a total charge in the superconductor that is given by

$$
\int d \varepsilon\left(\delta\left[j^{e}\right]_{d_{2}}+\delta\left[j^{e}\right]_{-d_{1}}\right)=0
$$

in the notations of Eq. (27). The charge conservation condition in this form can be regarded as the equation on the imbalance $\mu$. A finite supercurrent $J_{s}$ in the loop leads to a non-zero imbalance, which may act as a source of an electron-hole symmetry violation in the system. In the analogy with the Pethick-Smith effect, $\frac{29}{9}$ the imbalance defined by Eq. (36) is proportional to the scalar product of the temperature gradient and the supercurrent. We will see below that $\mu=A\left(T_{1}-T_{2}\right) \sin 2 \pi \Phi / \Phi_{0}$, where $A$ is a dimensionless coefficient that is mainly determined by the ratio of the Thouless energy $E_{c}=D / L^{2}$ to the mean temperature $T=\left(T_{1}+T_{2}\right) / 2$, such that $A$ is maximal for $T \sim E_{c}$.

From Eq. (36) we express the current in the superconducting wire as

$$
J_{s}=\frac{\sigma}{16 e} \int d \varepsilon\left(\delta\left[j^{e}\right]_{d_{2}}-\delta\left[j^{e}\right]_{-d_{1}}\right)
$$

where $\sigma$ is the Drude conductivity given by Eq. (20). Furthermore, the spacial integration of the electric current along the normal-metal wire gives

$$
J_{0}=\frac{d}{L} J_{s}^{e}+\frac{1}{L} \int_{-L_{1}}^{L_{2}} d x \frac{\sigma}{8 e} \int d \varepsilon j^{e} .
$$

Thus, the boundary condition (30) at the $N S$ interfaces together with Eqs. (11/36/37/38) describe the kinetic part of the problem. 
Let us now perform the calculation of $J_{0}$ to the second order in the parameters $\alpha_{1,2}$. In this approximation the anomalous components of the Green's function, $f^{R(A)}$, are found from the linearized Usadel equation (25) with the help of the boundary conditions (35) and

$$
\delta\left[\frac{d f^{R}}{d x}\right]_{-d_{1}}=-\alpha_{1} e^{i \chi_{1}}, \quad \delta\left[\frac{d f^{R}}{d x}\right]_{d_{2}}=-\alpha_{2} e^{i \chi_{2}},
$$

where the coefficients $\alpha_{1}$ and $\alpha_{2}$ introduced in the previous Section are determined by the $N S$ interface transparency. The conditions (39) follow directly from the retarded component of Eq. (28). With the help of the following notations

$$
\begin{aligned}
F_{a} & =\alpha_{a}^{2} \frac{\sinh z \ell_{a} \sinh z\left(L-\ell_{a}\right)}{z L \sinh z L}, \\
F_{12} & =\alpha_{1} \alpha_{2} \frac{\sinh z \ell_{1} \sinh z \ell_{2}}{z L \sinh z L},
\end{aligned}
$$

we express the anomalous Green's function near the $N S$ interfaces as

$$
\begin{aligned}
\frac{\alpha_{1}}{L} f^{R}\left(-d_{1}\right) & =e^{i \chi_{1}} F_{1}+e^{i \chi_{2}} F_{12}, \\
\frac{\alpha_{2}}{L} f^{R}\left(d_{2}\right) & =e^{i \chi_{2}} F_{2}+e^{i \chi_{1}} F_{12},
\end{aligned}
$$

and find the supercurrent density in the normal-metal wire as

$$
W=\left\{\begin{array}{cc}
W_{0} & x \in\left(-d_{1}, d_{2}\right), \\
0 & x \notin\left(-d_{1}, d_{2}\right),
\end{array}\right.
$$

where $W_{0}=-4 L \sin \varphi \operatorname{Im} F_{12}$. From Eq. (14a) we obtain

$$
\begin{gathered}
\delta\left[j^{e}\right]_{-d_{1}}=h\left(-d_{1}\right) W_{0}+4 \delta\left[\frac{d h_{\sigma}}{d x}\right]_{-d_{1}}, \\
\delta\left[j^{e}\right]_{d_{2}}=-h\left(d_{2}\right) W_{0}+4 \delta\left[\frac{d h_{\sigma}}{d x}\right]_{d_{2}},
\end{gathered}
$$

to the second order in $\alpha_{1,2}$.

The charge conservation condition (36), which determines the voltage difference $\mu / e$ between the superconductor and the normal-metal wire, can be written as

$$
\frac{d}{L} \int d \varepsilon h_{T} W_{0}=-8 \int d \varepsilon\left\{\delta\left[\frac{d h_{\sigma}}{d x}\right]_{d_{2}}+\delta\left[\frac{d h_{\sigma}}{d x}\right]_{-d_{1}}\right\} .
$$

The left-hand side of this expression is proportional to the temperature gradient due to the distribution function $h_{T}$. It is also antisymmetric with respect to the order parameter phase difference $\varphi=\chi_{1}-\chi_{2}=2 \pi \Phi / \Phi_{0}$ due to $W_{0}$. The righthand side of Eq. (44) is proportional to the imbalance $\mu$. We, therefore, come to the conclusion that the steady-state imbalance is an odd function of the temperature gradient and the applied flux.

From Eq. (37) we readily find the supercurrent

$$
\begin{aligned}
J_{s} & =\frac{\sigma}{8 e} \int d \varepsilon\left\{-\frac{1}{4}\left(h_{0}+\frac{\ell_{2}-\ell_{1}}{L} h_{T}\right) W_{0}\right. \\
& \left.+2\left(\delta\left[\frac{d h_{\sigma}}{d x}\right]_{d_{2}}-\delta\left[\frac{d h_{\sigma}}{d x}\right]_{-d_{1}}\right)\right\} .
\end{aligned}
$$

We also simplify Eq. (38) by calculating the spatial integral from the first term in the right-hand side of Eq. (14a) to the leading order in $\alpha$

$$
\frac{1}{L} \int_{-L_{1}}^{L_{2}} h W d x=\frac{d}{4 L}\left(h_{0}+\frac{\ell_{2}-\ell_{1}}{L} h_{T}\right) W_{0} .
$$

Thus, we obtain

$$
\begin{aligned}
J_{0} & =\frac{\sigma}{8 e} \int d \varepsilon\left\{\frac{2 d}{L}\left(\delta\left[\frac{d h_{\sigma}}{d x}\right]_{d_{2}}-\delta\left[\frac{d h_{\sigma}}{d x}\right]_{-d_{1}}\right)\right. \\
& \left.+\frac{h_{T}}{2 L} \frac{1}{L} \int_{-L_{1}}^{L_{2}} d x U-\frac{h_{\mu T}}{2 L} \frac{1}{L} \int_{-L_{1}}^{L_{2}} d x M_{+}\right\},
\end{aligned}
$$

where the spatial derivatives of $h$ and $h_{\sigma}$ are found from Eqs. (17).

The functions $h_{\mu}$ and $h_{\mu T}$ introduced in Eq. (18) are symmetric with respect to the energy $\varepsilon$ and antisymmetric with respect to the imbalance $\mu$, while $h_{0}$ and $h_{T}$ are antisymmetric in $\varepsilon$ and symmetric in $\mu$. Moreover, the last term in Eq. (47) can be disregarded in the linear response analysis. Indeed, the distribution function $h_{\mu T}$ is of a second order in the temperature gradient due to the fact that $\mu$ found from Eq. (44) is itself proportional to the temperature gradient. We shall, therefore, omit all terms containing $h_{\mu T}$ in our analysis of the thermopower for the parallelogram interferometer. We note, however, that the last term in Eq. (47) is the only one that changes sign with increasing temperature. We, therefore, regard the sign-reversal behavior of the thermoelectric response of the parallelogram interferometer observed by Parsons et $a l . \underline{16}$ as the second order effect that is beyond the linear response. Unlike other terms in Eq. (47) the last one is finite for a left-right symmetric setup and can easily dominate an experimental measurement for any finite temperature gradient. We postpone the detailed discussion of this contribution to the next Section.

Omitting the terms containing $h_{\mu T}$ we find that the discontinuity of the derivative $d h_{\sigma} / d x$ near the $N S$ interfaces is determined by the boundary conditions $(30)$ as

$$
\begin{gathered}
\delta\left[\frac{d h_{\sigma}}{d x}\right]_{-d_{1}}=\frac{L}{4} h_{\mu} \operatorname{Re}\left(F_{1}+F_{12} \cos \varphi\right), \\
\delta\left[\frac{d h_{\sigma}}{d x}\right]_{d_{2}}=\frac{L}{4} h_{\mu} \operatorname{Re}\left(F_{2}+F_{12} \cos \varphi\right),
\end{gathered}
$$

where we take advantage of the condition $h_{S \sigma}=0$.

The spatial integrals in Eq. (47) can be expressed through the anomalous Green's functions $f^{R, A}$ at the $N S$ interfaces with the help of Eq. (25) and the integration by parts. With the help of Eqs. 39411) we obtain

$$
\frac{\varepsilon}{D} \int_{-L_{1}}^{L_{2}} d x f^{R} \bar{f}^{A}=L \operatorname{Im}\left(\frac{F_{1}+F_{2}}{2}+F_{12} \cos \varphi\right),
$$

that leads to

$$
\frac{1}{L} \int_{-L_{1}}^{L_{2}} d x\left(f^{R} \bar{f}^{A}-f^{A} \bar{f}^{R}\right)=0 .
$$






FIG. 4: (Color online) The function $Y\left(T / E_{c}, d / L, \pi / 2\right)$ from Eq. 61) versus the ratio $T / E_{c}$ for the parallelogram interferometer with the symmetric arms $\ell_{1}=\ell_{2}$. The thermopower is maximal for $T \sim 2 E_{c}$, where $E_{c}=D / L^{2}$ is the Thouless energy associated with the normal-metal wire $N$. At high temperatures the thermopower decays according to the stretched exponential law (60) that is determined by the Thouless energy $E_{c}^{\prime}=D / d^{2}$ associated with the distance between the $N S$ junctions.

Thus, the second term in Eq. 477 is also vanishing. We will see in the next Section that this term is responsible for the particle-hole interference contribution to the thermopower that can be finite in the house interferometer. Finally, the first term in Eq. (47) gives rise to the only non-vanishing contribution to the current $J_{0}$ that is linear with respect to the temperature gradient.

From Eqs. (48) we obtain

$$
J_{0}=-\frac{\sigma d}{8 e} \int d \varepsilon h_{\mu} \operatorname{Re} \frac{F_{1}-F_{2}}{2},
$$

therefore the quasiclassical thermoelectric effect is absent in the left-right symmetric device $F_{1}=F_{2}$ in accordance with Ref. 22 and general symmetry considerations. The thermopower is readily found from the relation $S=\left(J_{0} / \sigma\right)\left(T_{2}-\right.$ $\left.T_{1}\right)^{-1}$, where we disregard the corrections to the Drude conductivity $\sigma$ arising from the proximity effect. This approximation is applicable to the second order in the $N S$ interface transparency.

In order to calculate $J_{0}$ in Eq. (51) we substitute $\mu$ found from Eq. (44) into Eq. (51). Disregarding the terms that are proportional to $h_{\mu T}$ we obtain the equation on the imbalance $\mu$ in the following form

$$
\begin{aligned}
\sin & \varphi \frac{d}{L} \int d \varepsilon h_{T} \operatorname{Im} F_{12} \\
& =\int d \varepsilon h_{\mu} \operatorname{Re}\left(\frac{1}{2}\left(F_{1}+F_{2}\right)+F_{12} \cos \varphi\right) .
\end{aligned}
$$

Expanding this equation to the first order in $\mu$ and the temperature gradient we obtain

$$
\mu=A\left(T_{1}-T_{2}\right) \sin \varphi
$$

where $T=\left(T_{1}+T_{2}\right) / 2$ is the mean temperature in the system and the coefficient $A$ is given by

$$
A=\frac{(d / L) \int d \varepsilon \varepsilon \cosh ^{-2} \frac{\varepsilon}{2 T} \operatorname{Im} F_{12}}{T \int d \varepsilon \cosh ^{-2} \frac{\varepsilon}{2 T} \operatorname{Re}\left(F_{1}+F_{2}+2 F_{12} \cos \varphi\right)} .
$$

We note that the coefficient $A$ is finite even for vanishing interface transparency parameters $\alpha_{1,2}$. From Eqs. (51153) we find the thermopower

$$
S=\frac{A d \sin \varphi}{8 e T} \int d \varepsilon \cosh ^{-2} \frac{\varepsilon}{2 T} \operatorname{Re}\left(F_{2}-F_{1}\right),
$$

that behaves roughly as $\sin \varphi$. The thermopower is finite only if there is a difference between $F_{1}$ and $F_{2}$, hence the device asymmetry is required. The Equation (55) describes the ana$\log$ of the Pethick-Smith effect in a superconducting proximity system.

The imbalance given by Eq. (53) is a non-monotonous function of the mean temperature $T$ that reaches its maximal value at $T \sim E_{c}=D / L^{2}$ and decays as a stretched exponent for $T \gg E_{c}^{\prime}=D / d^{2}$. It is worth noting that the imbalance itself is finite even for a left-right symmetric setup and in the limit of small transparency of the $N S$ interfaces. The temperature dependence of $\mu$ is determined by that of the coefficient $A$ in Eq. (54). The energy integrals in Eq. (54) can be rewritten as sums over the Matsubara frequencies. This greatly simplifies the calculation of the asymptotic behavior of $A$ at large temperatures.

Let us estimate the coefficient $A$ for a symmetric setup with $\ell_{1}=\ell_{2}=\ell$ and $\alpha_{1}=\alpha_{2}$. In the low temperature limit $T \ll E_{c}$ we obtain

$$
A=c_{\varphi} \frac{T L \ell}{D}, \quad c_{\varphi}=\frac{\pi^{2}}{18} \frac{1-2(\ell / L)^{2}}{1-(2 \ell / L)^{2} \sin ^{2}(\varphi / 2)} .
$$

For high temperatures $T \gg E_{c}^{\prime}$ we find from the Matsubara representation of Eq. (54) that

$$
A=c_{1} \frac{d}{\ell}\left(\sqrt{2 \pi T / E_{c}^{\prime}}-1\right) e^{-\sqrt{2 \pi T / E_{c}^{\prime}}}
$$

where

$$
c_{1}=\frac{\pi}{\left(2-2^{-1 / 2}\right) \zeta(3 / 2)} \approx 0.93 .
$$

The analysis shows that the coefficient $A$ reaches its maximal value at $T \simeq E_{c}=D / L^{2}$.

For $T \gg E_{c}^{\prime}$ the energy integral in Eq. (55) is decaying as $T^{-1 / 2}$, provided $\alpha_{1} \neq \alpha_{2}$. This decay crosses over to a stretched exponential decay for $\alpha_{1}=\alpha_{2}$ if the asymmetry is caused merely by a difference between $\ell_{1}$ and $\ell_{2}$. Therefore an asymmetry in the transparency parameter is of a greater importance to the thermopower than that in the arm lengths. For an equal arm interferometer $\ell_{1}=\ell_{2}=\ell$ with $\alpha_{1} \neq \alpha_{2}$ we obtain from Eqs. 56/57) in the limit $T \ll E_{c}=D / L^{2}$ that

$$
S=\frac{d c_{\varphi}\left(\alpha_{2}^{2}-\alpha_{1}^{2}\right) \sin \varphi}{4 e}\left(1+\frac{d}{L}\right) \frac{T \ell^{2}}{D} .
$$


For high temperatures, $T \gg E_{c}^{\prime}=D / d^{2}$, we get

$$
S=\frac{\pi d\left(\alpha_{2}^{2}-\alpha_{1}^{2}\right) \sin \varphi}{8 e} \frac{d^{2}}{L^{2}}\left(1-\sqrt{\frac{E_{c}^{\prime}}{2 \pi T}}\right) e^{-\sqrt{2 \pi T / E_{c}^{\prime}}} .
$$

The stretched exponential decay of the Pethick-Smith thermopower at high temperatures is entirely due to the behavior of the imbalance $\mu$.

For the interferometer with $\ell_{1}=\ell_{2}=\ell$ it is convenient to introduce the dimensionless function $Y$ by the following relation

$$
S=\frac{\pi d\left(\alpha_{2}^{2}-\alpha_{1}^{2}\right) \sin \phi}{8 e} Y\left(\frac{T}{E_{c}}, \frac{d}{L}, \varphi\right) .
$$

We see from Eqs. (78|60) that $Y$ is a very smooth function of the order parameter phase difference $\varphi$. The dependence on $\varphi$ disappears in the limit $T \gg E_{c}^{\prime}$. We plot the temperature dependence of $Y$ in Fig. 4 for $\varphi=\pi / 2$ and $d / L=1 / 4,1 / 3,1 / 2,2 / 3,3 / 4$. The thermopower reaches its maximal value in the interferometer with $\ell_{1}=\ell_{2}=d=L / 3$ at the temperature $T \simeq 2 E_{c}$. Our results are consistent with the experiment of Ref. 12 and with the numerical analysis of Refs. 34, 35, 40. The magnetic flux dependence of the thermopower is always antisymmetric and is essentially given by $\sin 2 \pi \Phi / \Phi_{0}$. A small deviation from this law may arise at low temperatures $T \lesssim E_{c}^{\prime}$. The strength of the proximity induced thermoelectric effect in the parallelogram interferometer is restricted by the asymmetry parameter $\alpha_{1}^{2}-\alpha_{2}^{2}$ and by the maximal value of the dimensionless function $Y$ that is approximated by $Y_{\max } \approx 0.05$.

\section{THERMOELECTRIC EFFECT IN THE HOUSE INTERFEROMETER}

In this Section we apply the same theory to the house interferometer depicted in Fig. 5. In our description of the system we introduce two normal-metal wires, $N$ and $N^{\prime}$, that are connected via a single tunnel junction with the transparency parameter $\alpha$. In the actual experiment of Ref. 12 the junction $N N^{\prime}$ is ballistic because the metallic wires are produced in a single lithography circle. We, however, expect that the symmetry of the thermoelectric coefficient is not affected by the detailed characteristics of the $N N^{\prime}$ junction.

Different space variables $x$ and $y$ are introduced as the coordinates along the wires $N$ and $N^{\prime}$, correspondingly. The proximity effect is described by the Green's functions $\check{g}_{c}(y)$ in $N^{\prime}$ and $\check{g}(x)$ in $N$. The $N N^{\prime}$ junction is located at $x=y=0$ and the wire $N^{\prime}$ is contacted to the superconductor at $y=-d_{1}$ and $y=d_{2}$. The transparency parameters $\tilde{\alpha}_{1}$ and $\tilde{\alpha}_{2}$ of the $N^{\prime} S$ interfaces are introduced in the same way as in the previous Section.

The thermopower measurements of Ref. 12 are shown in Fig. 1 $1 \mathrm{~b}$. In the experiment the distance between the $N^{\prime} S$ is given by $d=d_{1}+d_{2} \sim 2 \mu \mathrm{m}$ that is smaller than the phase coherence length $L_{\phi} \sim 4.6 \mu \mathrm{m}$ at $T=38 \mathrm{mK}$. We, therefore, expect that the setup is adequately described by the Usadel equation.

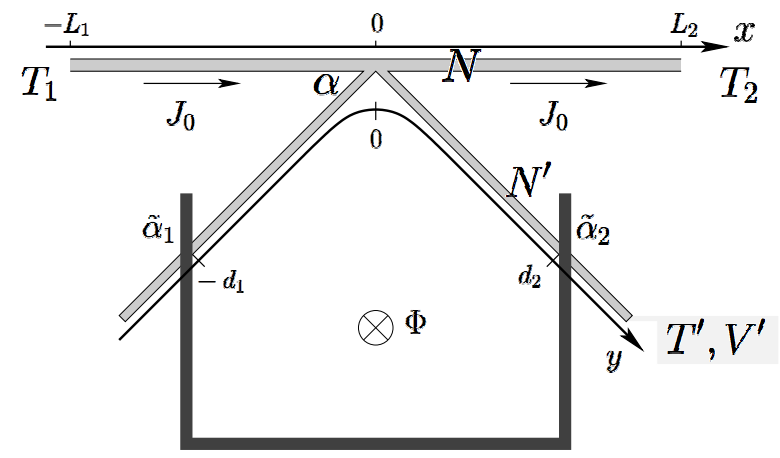

FIG. 5: The house interferometer. The wires $N$ and $N^{\prime}$ are normal metal wires. The dark wire is superconducting. The transparency parameters $\alpha, \tilde{\alpha}_{1,2}$ are defined by the ratio between the transmission probability per channel and the effective barrier length. Both symmetric and antisymmetric dependence of the thermopower on the magnetic flux $\Phi$ is possible in this setup. The antisymmetric effect, however, requires $d_{1} \neq d_{2}$. A charge imbalance between the superconducting wire and the temperature reservoirs is necessary for the symmetric thermopower.

The charge conservation condition in the loop formed by the superconductor and the $N^{\prime}$ wire is expressed as

$$
\delta\left[j^{e}\right]_{0}=0 .
$$

Thus, there exists no Pethick-Smith like contribution to $J_{0}$ that is analogous to the first term in Eq. (47). Another words the mechanism of the superconductor charging due to a supercurrent flow is absent in the house interferometer. Unlike in the parallelogram interferometer, the other two contributions to $J_{0}$ in Eq. (47) survive,

$$
J_{0}=\frac{\sigma}{8 e} \int d \varepsilon\left\{\frac{h_{T}}{2 L} \frac{1}{L} \int_{-L_{1}}^{L_{2}} d x Y-\frac{h_{\mu T}}{2 L} \frac{1}{L} \int_{-L_{1}}^{L_{2}} d x M_{+}\right\} .
$$

It is worth noting that the expression (63) for the dissipative current between the temperature reservoirs is valid for any interface transparency.

The first term of Eq. (63), which is proportional to $h_{T}$, is antisymmetric in the magnetic field and is further referred to as the interference contribution. This contribution is insensitive to the imbalance and vanishes in the parallelogram interferometer because of the property (50). It remains, however, finite in the house interferometer where the length of the interfering trajectories is fixed by the distances $d_{1}$ and $d_{2}$ between the $N^{\prime} S$ interfaces and the $N N^{\prime}$ junction.

Two interfering quasiparticle trajectories, which contribute to the heat transfer between the reservoirs, are depicted schematically in Fig. 6. The scattering amplitude of the process can be written as

$$
\mathcal{A}_{\varepsilon} \sim \alpha^{2}\left|\tilde{\alpha}_{1} e^{i\left(k_{e}-k_{h}\right) d_{1}+i \chi_{1}}+\tilde{\alpha}_{2} e^{i\left(k_{e}-k_{h}\right) d_{2}+i \chi_{2}}\right|^{2},
$$

where $k_{e, h}=k_{F} \pm \varepsilon / v_{F}$ are the wave vectors of electrons and holes. Here we took into account that the Andreev reflection 


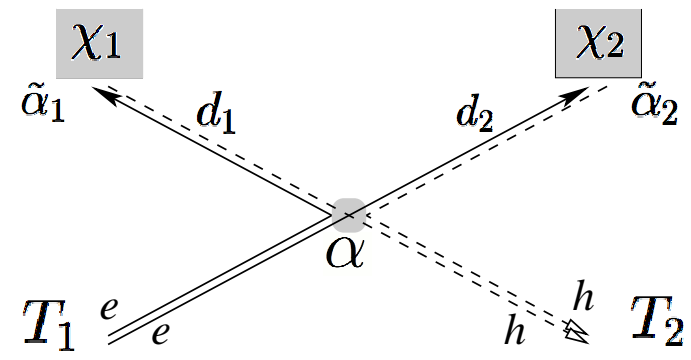

FIG. 6: An example of interfering trajectories that contribute to the thermoelectric response of the house interferometer.

process is of a second order in the $N^{\prime} S$ interface transparency. The corresponding contribution to the thermopower is proportional to the energy derivative of the scattering amplitude $\mathcal{A}_{\varepsilon}$ at the Fermi energy,

$$
\left.S \sim \frac{d \mathcal{A}_{\varepsilon}}{d \varepsilon}\right|_{\varepsilon=0} \propto \alpha^{2} \tilde{\alpha}_{1} \tilde{\alpha}_{2}\left(d_{2}-d_{1}\right) \sin \varphi
$$

which is a manifestly antisymmetric function of the magnetic flux. The result of Eq. (65) is finite if $d_{1} \neq d_{2}$, while the difference in the transmission parameters $\tilde{\alpha}_{1}$ and $\tilde{\alpha}_{2}$ is neither necessary nor sufficient for the observation of the interference effect. Integration over all possible trajectories in the parallelogram interferometer lead to the complete suppression of interference that is reflected in Eq. 50.

The interference contribution to the thermopower discussed above has not been observed in experiment, because it is overwhelmed by the second term in Eq. (63) which we refer to as the symmetric contribution. This term is proportional to $h_{\mu T}$ and symmetric with respect to the magnetic flux provided $\mu$ is flux independent. It remains to be finite for a left-right symmetric device but vanishes for $\mu=0$.

The experimental measurements of Ref. 12 shown in Fig. 1 1 b can be regarded as demonstration of a finite imbalance state formed in the house interferometer. The experiment suggests that a constant imbalance $\mu$ is largely independent on the temperature gradient in the wire $N$ as well as on the supercurrent in the loop. Since $\mu=0$ is the only solution to the charge conservation condition (62), the origin of such a steady state is not entirely clear. We stress, however, that a tiny charge imbalance can originate in the usual thermoelectric effect provided $N^{\prime}$ wire is in an equilibrium state with a temperature $T^{\prime}$ that is smaller than both $T_{1}$ and $T_{2}$ due to the phonon cooling. In this case we can estimate $\mu=e\left(T-T^{\prime}\right) S_{N^{\prime}}$, where $S_{N^{\prime}}$ is the small thermopower of the $N^{\prime}$ wire. The tiny charge imbalance $\mu$ will be seen to have a great effect on the thermopower due the proximity effect enhancement factor. In what follows we simply keep $\mu$ as a phenomenological parameter of the quasiclassical theory.

We start by calculating the anomalous component $f^{R}(x)$ of the Green's function in the normal metal wire $N$. We again take advantage of the linearized Usadel equation (25) and employ the rigid boundary condition

$$
\delta\left[\frac{d f^{R}}{d x}\right]_{0}=-\alpha f_{c 0}^{R}
$$

where $f_{c 0}^{R}=f_{c}^{R}(y=0)$ is the value of the anomalous Green's function at the junction.

In addition to Eq. (66) we use Eq. (35) and the continuity of $f^{R}(x)$ at $x=0$. As the result we obtain

$f^{R}=\frac{\alpha f_{c 0}^{R}}{z \sinh z L}\left\{\begin{array}{lr}\sinh z L_{2} \sinh z\left(L_{1}+x\right), & -L_{1}<x<0 \\ \sinh z L_{1} \sinh z\left(L_{2}-x\right), & 0<x<L_{2}\end{array}\right.$.

We calculate the spatial integrals in Eq. 63) with the help of Eq. 67) as

$$
\begin{aligned}
& \frac{1}{L} \int_{-L_{1}}^{L_{2}} d x\left(f^{R} \bar{f}^{A} \pm f^{A} \bar{f}^{R}\right)=\frac{D}{2 \varepsilon}\left(f_{c 0}^{R} \bar{f}_{c 0}^{A} \pm f_{c 0}^{A} \bar{f}_{c 0}^{R}\right) \operatorname{Im} F \\
& F=\alpha^{2} \frac{\sinh z L_{1} \sinh z L_{2}}{z L \sinh z L}, \quad L=L_{1}+L_{2} .
\end{aligned}
$$

It is worth noting that the first term of Eq. 63) is not necessarily vanishing unlike in the parallelogram interferometer.

We evaluate the second term of Eq. (63) to the leading order in the $N^{\prime} S$ interface transparency by calculating the following integral

$$
\begin{aligned}
& \frac{1}{L} \int_{-L_{1}}^{L_{2}} d x\left(f^{R} \bar{f}^{R}+f^{A} \bar{f}^{A}\right)=\frac{D}{2 \varepsilon} \operatorname{Im} f_{c 0}^{R} \bar{f}_{c 0}^{R}(K-F), \\
& K=\alpha^{2} \frac{L_{1} \sinh ^{2} z L_{2}+L_{2} \sinh ^{2} z L_{1}}{L \sinh ^{2} z L}
\end{aligned}
$$

that is also expressed through the the anomalous Green's function in the $N^{\prime}$ wire. Thus, from Eq. 63) we find, to the second order in $f_{c 0}$, that

$$
\begin{aligned}
& J_{0}=\frac{\sigma}{8 e} \int d \varepsilon \frac{D}{2 \varepsilon}\left\{\frac{h_{T}}{2 L}\left(f_{c 0}^{R} \bar{f}_{c 0}^{A}-f_{c 0}^{A} \bar{f}_{c 0}^{R}\right) \operatorname{Im} F\right. \\
& \left.-\frac{h_{\mu T}}{2 L}\left(\left(f_{c 0}^{R} \bar{f}_{c 0}^{A}+f_{c 0}^{A} \bar{f}_{c 0}^{R}\right) \operatorname{Im} F+\operatorname{Im} f_{c 0}^{R} \bar{f}_{c 0}^{R}(F-K)\right)\right\} .
\end{aligned}
$$

It remains to determine the function $f_{c}(y)$ from the Usadel equation in the wire $N^{\prime}$. Since we calculate the current $J_{0}$ to the leading order in the interface transparency parameters the tunneling between $N$ and $N^{\prime}$ can be disregarded in the Usadel equation. Taking advantage of the rigid boundary conditions in the form

$$
\left.\frac{d f_{c}^{R}}{d y}\right|_{-d_{1}}=-\tilde{\alpha}_{1} e^{i \chi_{1}},\left.\quad \frac{d f_{c}^{R}}{d y}\right|_{d_{2}}=\tilde{\alpha}_{2} e^{i \chi_{2}},
$$

we obtain

$$
f_{c}^{R}(y)=\tilde{\alpha}_{1} e^{i \chi_{1}} \frac{\cosh z\left(d_{2}-y\right)}{z \sinh z d}+\tilde{\alpha}_{2} e^{i \chi_{2}} \frac{\cosh z\left(d_{1}+y\right)}{z \sinh z d},
$$

where $d=d_{1}+d_{2}$. Thus, the value of the anomalous Green's function at the contact is given by

$$
f_{c 0}^{R}=\tilde{\alpha}_{1} e^{i \chi_{1}} \frac{\cosh z d_{2}}{z \sinh z d}+\tilde{\alpha}_{2} e^{i \chi_{2}} \frac{\cosh z d_{1}}{z \sinh z d} .
$$

Substitution of Eq. (73) to Eq. (70) completes the calculation of the thermoelectric effect. 
Let us now analyze two different terms in Eq. (70) separately. We first let $\mu=0$ so that $h_{\mu T}=0$ and the only contribution to Eq. (70) is antisymmetric in the magnetic flux. From Eq. (73) we find the expression

$$
f_{c 0}^{R} \bar{f}_{c 0}^{A}-f_{c 0}^{A} \bar{f}_{c 0}^{R}=4 \tilde{\alpha}_{1} \tilde{\alpha}_{2} \sin \varphi \frac{\operatorname{Im}\left(\cosh z d_{1} \cosh z^{*} d_{2}\right)}{|z \sinh z d|^{2}} .
$$

In order to obtain the thermopower $S=\left(J_{0} / \sigma\right)\left(T_{2}-T_{1}\right)^{-1}$ we substitute Eq. (74) to Eq. (70) and expand $h_{T}$ to the linear order in the temperature gradient as

$$
h_{T}=-\frac{\varepsilon\left(T_{1}-T_{2}\right)}{T^{2} \cosh ^{2} \frac{\varepsilon}{2 T}} .
$$

The resulting expression for $S$ reads

$$
S=\frac{\alpha^{2} \tilde{\alpha}_{1} \tilde{\alpha}_{2} \sin \varphi D d^{2}}{8 e L} \frac{B}{T}
$$

with the coefficient $B$ given by

$$
B=\int d \varepsilon \frac{\operatorname{Im}\left(\cosh z d_{1} \cosh z^{*} d_{2}\right)}{|z d \sinh z d|^{2} T \cosh ^{2} \frac{\varepsilon}{2 T}} \operatorname{Im} \frac{\tanh (z L / 2)}{z L \sinh z L},
$$

where we let $L_{1}=L_{2}=L / 2$ for simplicity. The Equation (76) describes the interference contribution to the thermopower. The result of Eq. (76) is indeed proportional to $\alpha^{2} \tilde{\alpha}_{1} \tilde{\alpha}_{2} \sin \varphi$ and is vanishing for $d_{1}=d_{2}$ in accordance with the estimate (65). Moreover, in the limit $T \ll \min \left\{E_{c}, E_{c}^{\prime}\right\}$, where $E_{c}=D / L^{2}$ and $E_{c}^{\prime}=D / d^{2}$, we find

$$
S=\frac{\alpha^{2} \tilde{\alpha}_{1} \tilde{\alpha}_{2}\left(d_{2}-d_{1}\right) D L}{198 e T d} \sin \varphi,
$$

that is equivalent to Eq. 65]. We note that the divergence of Eq. (78) in the limit $T \rightarrow 0$ is regularized by the high order processes in the parameter $\alpha$. The result of Eq. (78) is, therefore, valid only for $T \gtrsim \alpha^{2} D$. More accurate expression can be obtained by solving the non-linear Usadel equation in the normal-metal wire $N^{\prime}$.

For higher temperatures $T \gg \max \left\{E_{c}, E_{c}^{\prime}\right\}$ it is legitimate to substitute $\cosh (\varepsilon / 2 T) \rightarrow 1$ in Eq. (77). The remaining integral gives the dependence of the thermopower on the distances $L, d_{1}$, and $d_{2}$. Thus, unlike the Pethick-Smith contribution (61) to the thermopower, the interference contribution has a monotonous temperature dependence and decays as the power law $T^{-2}$ at high temperatures. It is also the only contribution that remains finite for $\mu=0$.

Let us consider now the symmetric contribution to Eq. (70) that emerges for a finite $\mu$. Since this contribution does not rely on the device asymmetry, we let for simplicity $d_{1}=d_{2}=$ $d / 2$. Following the discussion above we treat the imbalance $\mu$ as a constant in our quasiclassical analysis and calculate the thermopower with the assumption that $\mu$ is independent on the temperature gradient $T_{2}-T_{1}$. The substitution of Eq. (73) to Eq. (70) gives

$$
J_{0}=-\frac{\sigma}{4 e} \frac{D\left(\tilde{\alpha}_{1}^{2}+\tilde{\alpha}_{2}^{2}+2 \tilde{\alpha}_{1} \tilde{\alpha}_{2} \cos \varphi\right)}{T L} \int d \varepsilon h_{\mu T} P,
$$

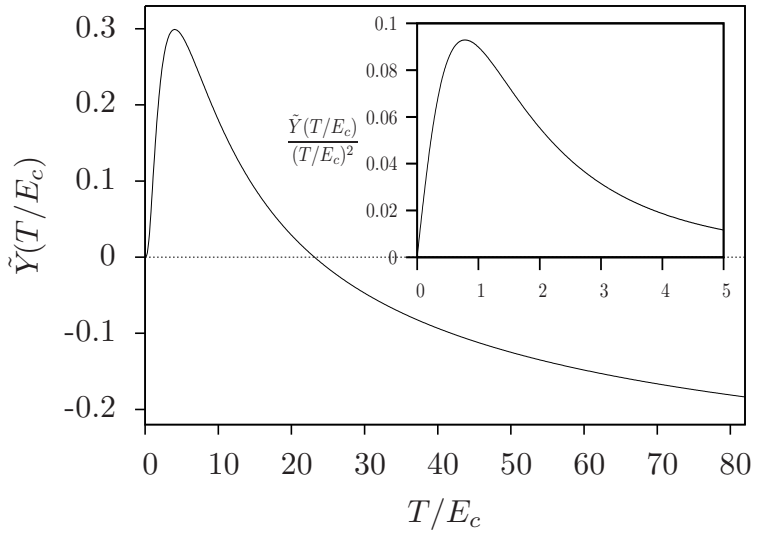

FIG. 7: The function $\tilde{Y}\left(T / E_{c}\right)$ from Eqs. $\left.87 / 88\right)$ versus the ratio $T / E_{c}$ for the house interferometer with $d_{1}=d_{2}$. The function is maximal for $T \approx 4 E_{c}$ and changes sign at $T \approx 24 E_{c}$, where $E_{c}=$ $D / L^{2}$ is the Thouless energy associated with the normal-metal wire $N$. The inset shows the temperature dependence of the thermopower in Eq. 87) under the assumption that the imbalance $\mu$ and the length $L$ are temperature independent. The thermopower is maximal at $T \approx$ $E_{c}$ and decays as $T^{-2}$ for $T \gg 24 E_{c}$.

where

$$
P=\frac{T}{16 \varepsilon} \operatorname{Im}\left\{\frac{F}{|z \sinh z d / 2|^{2}}+\frac{F-K}{2(z \sinh z d / 2)^{2}}\right\} .
$$

Thus, for the completely symmetric case $\tilde{\alpha}_{1}=\tilde{\alpha}_{2}$ the thermopower has an overall phase dependent factor $1+\cos \varphi$, that qualitatively agrees with the experimental curve shown in Fig. 1 $1 \mathrm{~b}$. In general, the minimal absolute value of the symmetric contribution to the thermopower is determined by the asymmetry parameter $\left(\tilde{\alpha}_{1}-\tilde{\alpha_{2}}\right)^{2}$.

We note, however, that the integral in Eq. (80) diverges at small energies. This divergence is regularized by the high order processes in the parameter $\alpha$. In order to get a well-defined expression we need to solve the non-linear Usadel equation in $N^{\prime}$ that is a daunting task. We give instead an analytical estimate to the symmetric thermopower by replacing the $N^{\prime}$ wire with a diffusive quantum dot. In this model the Green's function, $\check{g}_{c}$, in the dot can be obtained in the spirit of the Nazarov's circuit theory ${ }^{41}$ from the matrix current conservation condition

$$
\left[\tilde{\alpha}_{1} \hat{g}_{S, 1}^{R}+\tilde{\alpha}_{1} \hat{g}_{S, 2}^{R}+\alpha \sigma_{z}, g_{c}^{R}\right]=0 .
$$

Resolving the condition for $\hat{g}_{c}^{R}$ we obtain

$$
f_{c}^{R}=\left(\tilde{\alpha}_{1} e^{i \chi_{1}}+\tilde{\alpha}_{2} e^{i \chi_{2}}\right) u^{-1},
$$

with

$$
\begin{aligned}
u & =\left(\left(\tilde{\alpha}_{1}+\tilde{\alpha}_{2}\right)(-i \varepsilon / \Delta)+\alpha \sqrt{1+(-i \varepsilon / \Delta)^{2}}\right)^{2} \\
& +\tilde{\alpha}_{1}^{2}+\tilde{\alpha}_{2}^{2}+2 \tilde{\alpha}_{1} \tilde{\alpha}_{2} \cos \varphi .
\end{aligned}
$$

In this approximation the anomalous Green's function $f_{c}^{R}=$ $f_{c 0}^{R}$ acquires no spacial dependence on $y$. The function $f_{c}^{R}$ 
remains to be small in the parameters $\tilde{\alpha}_{1}$ and $\tilde{\alpha}_{2}$, therefore Eq. (70) is still a legitimate approximation for the thermopower to the leading order in $\tilde{\alpha}_{1,2}$. Substituting $f_{c 0}^{R}=f_{c}^{R}$ from Eq. (82) to Eq. (70) we arrive at the result (79) with

$$
P=\frac{T}{\varepsilon} \operatorname{Im}\left\{\frac{F}{|u|}+\frac{F-K}{2 u}\right\} .
$$

In order to be consistent we have to take the limit $\varepsilon \ll \Delta$ and $\tilde{\alpha}_{a} \ll \alpha$ in the expression (83), hence

$$
u=\alpha^{2},
$$

and the parameter $\alpha$ cancels out completely in Eq. (84). In the limit $\mu \ll T$ and $T_{1}-T_{2} \ll T$ we expand $h_{\mu T}$ as

$$
h_{\mu T} \simeq \frac{\mu\left(T_{1}-T_{2}\right)}{T^{2} \cosh ^{2} \varepsilon / 2 T}\left(1-\frac{\varepsilon}{T} \tanh \frac{\varepsilon}{2 T}\right)
$$

and substitute this expression into Eq. (79) with the function $P$ given by Eq. (84). This provides us with the final result for the thermopower $S=\left(J_{0} / \sigma\right)\left(T_{2}-T_{1}\right)^{-1}$ of the house interferomter

$$
S=-\frac{D \mu\left(\tilde{\alpha}_{1}^{2}+\tilde{\alpha}_{2}^{2}+2 \tilde{\alpha}_{1} \tilde{\alpha}_{2} \cos \varphi\right)}{4 e T^{2} L} \tilde{Y}\left(\frac{T}{E_{c}}\right),
$$

where $E_{c}=D / L^{2}$ and the dimensionless function $\tilde{Y}\left(T / E_{c}\right)$ is given by

$$
\tilde{Y}=\int_{-\infty}^{\infty} d x \frac{2 x \tanh x-1}{4 x \cosh ^{2} x} \operatorname{Im}\left\{\frac{3 \tanh \kappa_{x}}{2 \kappa_{x}}-\frac{1}{\cosh ^{2} \kappa_{x}}\right\},
$$

with $\kappa_{x} \equiv \sqrt{-i x T / E_{c}}$. In the limit $T \ll E_{c}$ we find $\tilde{Y}\left(T / E_{c}\right)=\left(187 \pi^{2} / 7560\right)\left(T / E_{c}\right)^{3}$. The function $\tilde{Y}$ determines the temperature dependence of the symmetric contribution to the thermopower (see Fig. 7) provided $\mu$ is a temperature independent constant. This contribution is not suppressed by any asymmetry factor unlike the Pethick-Smith thermopower (61) and the interference contribution (76). Moreover, the symmetric contribution is neither monotonous nor sign-definite function of temperature. At $T \sim E_{c}$ the thermopower approaches its maximum value, which can be estimated as

$$
\left.S\right|_{T \simeq E_{c}} \approx-0.1 \frac{\mu L^{3}}{4 e D}\left(\tilde{\alpha}_{1}^{2}+\tilde{\alpha}_{2}^{2}+2 \tilde{\alpha}_{1} \tilde{\alpha}_{2} \cos \varphi\right) .
$$

Thus, the presence of a very small imbalance $\mu$ leads to large observable magnetic flux dependence of the thermopower. The absolute value of the thermopower at $T \sim E_{c}$ and $\Phi=0$ is determined by the parameter $\mu L /\left(\ell_{B} E_{c}\right)$, where $\ell_{B}$ is the effective barrier length that is orders of magnitude smaller than $L$. This is the proximity effect enhancement factor that gives rise to an anomalously strong sensitivity of the thermopower to the charge imbalance $\mu$.

We also see from Eqs. (87/88) that the thermopower changes sign at $T \approx 24 E_{c}$ and decays as $T^{-2}$ at higher temperatures since $\tilde{Y} \rightarrow-2 / 5$ for $T \gg 24 E_{c}$. For higher temperatures the length $L$ has to be substituted by the phase coherence length $L_{\phi}$ provided the latter is smaller than the distance between the reservoirs. The temperature dependence of $L_{\phi}$ complicates the direct comparison to the experimental data. In particular this dependence should strongly enhance the reversed thermopower for $T \gtrsim 24 E_{c}$. Nevertheless, the sign reversal behavior of the the symmetric thermopower observed by Parsons et al. ${ }^{16,17}$ is in a qualitative agreement with the result of Eq. 877.

A thorough experimental test of the presented theory can be performed with the help of an independent experimental control over the imbalance $\mu$ that has a strong effect on the sign and the magnitude of the symmetric contribution to the thermopower in the house interferometer. For the parallelogram interferometer the symmetric contribution is described by the last term in Eq. (47), that is of the second order in the temperature gradient. This term can be roughly estimated by substituting $\mu$ from Eq. (53) to Eq. (87). This second order effect reverses sign with increasing temperature and is antisymmetric in $\Phi$ due to the magnetic flux dependence of the imbalance in the parallelogram interferometer. It is likely that the effect of this type has been observed in the experiments by Parsons et al $.16,17$

\section{CONCLUSION}

In this paper we applied quasiclassical theory to study the origin of the thermoelectric effects in Andreev interferometers. The theory predicts three additive contributions to the electric current expressed by Eqs. (11al14a) in the presence of the superconducting proximity effect. Depending on the geometry of Andreev interferometer and its parameters any of these contributions may dominate the quasiclassical thermoelectric response of the normal-metal wire. One can classify the observed thermoelectric effect by its dependence on the temperature and the magnetic flux piercing the interferometer. The last term in Eq. (14a), which describes the proximity induced supercurrent, and the second term, which is related to the interference contribution, are antisymmetric in the magnetic flux. Even though the supercurrent cannot flow between the normal metal reservoirs the last term in Eq. 114a can, nevertheless, contribute to the thermopower by means of the proximity-induced Pethick-Smith effect. This effect takes place provided the temperature gradient is aligned in a part of the normal metal wire with the supercurrent. This situation is realized in the parallelogram interferometer considered in the Section IV

The left-right asymmetry of the parallelogram interferometer is responsible for a difference in the dissipative charging currents flowing from the normal-metal reservoirs to the superconductor. As the result of this asymmetry a compensating dissipative current $J_{0}$ (51) is flowing between the normal-metal reservoirs. The analytical expression for the thermopower in the case of weak proximity effect is given by Eqs. (54|55). The proximity-induced Pethick-Smith effect demonstrates a non-monotonous temperature dependence with a maximum at $T \simeq 2 E_{c}$ (see Fig. (4) and is characterized 
by the stretched exponential decay for high temperatures $(60)$. The effect does not rely on the quasiparticle phase coherence in the normal-metal wire. The most important ingredient of the theory is the difference in the electron distribution function near the first and the second $N S$ interface in the parallelogram interferometer.

In contrast, the interference contribution to the thermopower, which is given by the second term in Eq. (14a), does require a phase coherence in the normal-metal wire. This contribution is the only one that exists in the absence of a charge imbalance between the superconductor and the normal metal. This effect cannot be seen in the parallelogram interferometer but might be observed in the house one provided $d_{1} \neq d_{2}$ in Fig. 5. The interference contribution is characterized by a monotonous temperature decay, that is estimated in Eqs. (76177) in the Section V

Finally, the first term in Eq. 14a) is regarded as the symmetric contribution. It is largely insensitive to the left-right symmetry of the device and gives rise to the thermopower that is symmetric with respect to the magnetic flux (with the assumption that $\mu$ is flux independent). In the Section $\nabla$ we argue that the existing experiments indicate the presence of such a steady state imbalance in the house interferometer. The symmetric contribution is characterized by a peculiar temperature dependence in Eqs. (87/88) that is neither monotonous nor sign-definite. This contribution strongly affects the thermoelectric response of the parallelogram interferometer in the second order with respect to the temperature gradient.

\section{Acknowledgments}

The author acknowledges discussions with W. Belzig, A. A. Golubov, and Yu. V. Nazarov and the kind hospitality of the Isaac Newton Institute for Mathematical Sciences of the Cambridge University, where this work has been partially written. The author thanks Venkat Chandrasekhar for reading the manuscript prior publication and is especially grateful to Igor Aleiner for numerous discussions and motivation of the present study.
1 A. A. Abrikosov, Introduction to the Theory of Normal Metals, New York, Academic Press Inc (1972).

2 N. F. Mott and H. Jones, The Theory of the Properties of Metals and Alloys, (Clarendon, Oxford, 1936).

3 V. L. Ginzburg, J. Phys. USSR 8, 148 (1944); [Zh. Eksp. i Teor. Fiz. 14, 177 (1944)].

${ }^{4}$ V. P. Steiner and P. Grassmann, Phys. Z. 36, 527 (1935).

5 R. P. Huebener, in Solid State Physics, edited by H. Ehrenreich, F. Seitz, and D. Turnbull (Academic Press, New York, 1972).

6 Yu. M. Galperin, V. L. Gurevich, and V. I. Kozub, JETP Lett. 17, 476 (1973); [Pis'ma v Zh. Eksp. i Teor. Fiz. 17, 687 (1973)].

7 J. C. Garland and D. J. Van Harlingen, Phys. Lett. A. 47, 423 (1974).

8 N. V. Zavaritskii, JETP Lett. 19, 126 (1974); [Pis'ma v Zh. Eksp. i Teor. Fiz. 19, 205 (1974)].

9 D. J. Van Harlingen, D. F. Heidel, and J. C. Garland, Phys. Rev. B. 21, 1842 (1980).

10 J. Koláček, P. Lipavský, Phys. Rev. B 71, 092503 (2005).

11 V. L. Gurevich, V. I. Kozub, and A. L. Shelankov, Eur. Phys. J. B 51, 285 (2006).

12 J. Eom, C.-J. Chien, and V. Chandrasekhar, Phys. Rev. Lett. 81, 437 (1998).

13 J. Eom, C.-J. Chien, and V. Chandrasekhar, Superlatt. and Microstruct. 25, 733 (1999).

14 J. Eom, C.-J. Chien, and V. Chandrasekhar, J. Low Temp. Phys. 118, 617 (2000).

15 D. A. Dikin, S. Jung, and V. Chandrasekhar, Europhys. Lett. 57, 564 (2002).

16 A. Parsons, I. A. Sosnin, and V. T. Petrashov, Phys. Rev. B 67, 140502(R) (2003).

17 A. Parsons, I. Sosnin, and V. T. Petrashov, Physica E 18, 316 (2003).

18 Z. Jiang and V. Chandrasekhar, Phys. Rev. Lett. 94, 147002 (2005).
19 Z. Jiang and V. Chandrasekhar, Phys. Rev. B 72, 020502(R) (2005)

20 Z. Jiang and V. Chandrasekhar, Chin. J. Phys. 43, 693 (2005).

${ }^{21}$ P. Cadden-Zimansky, Z. Jiang, and V. Chandrasekhar, New J. Phys. 9, 116 (2007).

22 R. Seviour and A. F. Volkov, Phys. Rev. B 62, R6116 (2000).

23 V. R. Kogan, V. V. Pavlovskii, and A. F. Volkov, Europhys. Lett. 59, 875 (2002).

${ }^{24}$ C. J. Pethick and H. Smith, J. Phys. C 13, 6313(1980).

25 T. J. Rieger, D. J. Scalapino, J. E. Mercereau, Phys. Rev. Lett. 27, 1787 (1971).

${ }^{26}$ M. L. Yu, J. E. Mercereau, Phys. Rev. Lett. 28, 1117 (1972).

27 J. Clarke, Phys. Rev. Lett. 28, 1363 (1972).

28 M. Tinkham and J. Clarke, Phys. Rev. Lett. 28, 1366 (1972).

29 C. J. Pethick and H. Smith, Phys. Rev. Lett. 43, 640 (1979).

30 J. Clarke, B. R. Fjordbøge, and P. E. Lindelof, Phys. Rev. Lett. 43, 642 (1979).

31 J. Clarke and M. Tinkham, Phys. Rev. Lett. 44, 106 (1980).

32 A. Schmid and G. Schön, Phys. Rev. Lett. 34, 941 (1975); 43, 793 (1979)

33 A. Schmid and G. Schön, J. Low Temp. Phys. 20, 207 (1975).

${ }^{34}$ P. Virtanen and T. T. Heikkilä, Phys. Rev. Lett. 92, 177004 (2004).

35 P. Virtanen and T. T. Heikkilä, J. Low Temp. Phys. 136, 401 (2004).

${ }^{36}$ P. Virtanen and T. T. Heikkilä, Phys. Rev. B 75, 104517 (2007).

37 V. Chandrasekhar, "The Physics of Superconductors", Vol II, edited by Bennemann and Ketterson, Springer-Verlag, (2004).

38 T. H. Stoof and Yu. V. Nazarov, Phys. Rev. B 53, 14496 (1996).

39 A. A. Golubov, M. Yu. Kupriyanov, E. Il'ichev, Rev. Mod. Phys. 76, 411 (2004).

40 A. F. Volkov and V. V. Pavlovskii, Phys. Rev. B. 72, 014529 (2005).

41 Y. V. Nazarov, Phys. Rev. Lett. 73, 1420 (1994). 